and

$$
W\left(r_{0}\right)=0 \quad \text { or } \quad 1 .
$$

Equation (16) is equivalent to a variational problem for the first eigenvalue of

$$
\lambda=\min \frac{\int_{n}^{\infty}\left(g^{\prime 2}-K g^{2}\right) d r}{\int_{0}^{\infty} g^{2} d r} .
$$

We must show that the ratio in Eq. (17) is negative for some function $g$ satisfying the boundary condition. Then $\lambda<0$ and the minimum will then be below this value. For the case of $W\left(r_{0}\right)=0, K$ will always be negative and therefore all characteristic numbers $\lambda$ are positive and no neutral oscillation occurs. In the case of $W\left(r_{0}\right)=1$ at $r_{0}=0$, $K$ may be positive, and there may exist a function such that the ratio in Eq. (17) is negative. Numerical calculations for this case are given in [Ref. 3].

It is interesting to note that the term

$$
\frac{1}{r}\left(W^{\prime \prime}-\frac{W^{\prime}}{r}\right)=\frac{d}{d r}\left(\frac{1}{r} \frac{d W}{d r}\right)
$$

is the gradient of $-\eta / r=r^{-1} W^{\prime}(r)$ where $\eta$ is the vorticity of the basic flow in accordance with the parallel flow assumption. Thus, the condition for neutral oscillation corresponds to the vanishing of this gradient at some point. This is somewhat analogous to the twodimensional case discussed by Lin in [Ref. 4] where a physical interpretation of this condition for stability is given. However, here the important quantity is $\eta / r$ and not the vorticity alone.

The extension of these discussions to three-dimensional disturbances should allow for the dependency of the disturbances on the anguiar variable $\varphi$.

\title{
REFerences
}

1. S. Goldstein, ed. Modern development in fuid dynamics, I, Oxford University Press, 1938

2. J. L. Synge, Hydrodynamic stability, Semi-centennial Publications, Am. Math. Soc. 2, 227-269 (1938)

3. H. G. Lew, Part IV of Hydrodynamic stability, Technical Report No. 2, Dept. of Aeronaut. Eng. The Pennsylvania State University, (1954)

4. C. C. Lin, On the stability of two-dimensional parallel flows, Part II, Quart. Appl. Math. 3, 218-234 (1945)

\section{ON SOURCE AND VORTEX OF FLUCTUATING STRENGTH TRAVELLING BENEATH A FREE SURFACE*}

By H. S. TAN (University of Notre Dame)

In the coordinate system which moves with the travelling source or vortex at constant forward speed $c$, and under the hypothesis that the resulting fluid motion is irrotational, one can define a disturbance velocity potential $\Phi(x, y, t)$ for the two dimensional fluid motion through the differential equation

*Received Oct. 18, 1954. Revised manuscript received Feb. 4, 1955. 


$$
\frac{\partial^{2} \Phi}{\partial x^{2}}+\frac{\partial^{2} \Phi}{\partial y^{2}}=0
$$

the boundary condition on free surface $Y(y=0)$

i.e.

$$
\begin{aligned}
& \frac{\partial \Phi}{\partial t}-c \frac{\partial \Phi}{\partial x}=-g Y, \\
& \frac{\partial Y}{\partial t}-c \frac{\partial Y}{\partial x}=\frac{\partial \Phi}{\partial y},
\end{aligned}
$$

$$
\frac{\partial^{2} \Phi}{\partial t^{2}}-2 c \frac{\partial^{2} \Phi}{\partial t \partial x}+c^{2} \frac{\partial^{2} \Phi}{\partial x^{2}}+g \frac{\partial \Phi}{\partial y}=0,
$$

and the physical condition that the upstream disturbance must vanish at infinity, i.e.

$$
\lim _{x \rightarrow \infty}\left[\left|\frac{\partial \Phi}{\partial x}\right|^{2}+\left|\frac{\partial \Phi}{\partial y}\right|^{2}\right]=0 \text {. }
$$

When the moving source or vortex executes harmonic fluctuation in strength, by further writing the velocity potential in the form

$$
\Phi(x, y, t)=\Re \phi(x, y) \exp (i \omega t),
$$

one obtains the following differential system for $\phi$ :

$$
\begin{gathered}
\frac{\partial^{2} \phi}{\partial x^{2}}+\frac{\partial^{2} \phi}{\partial y^{2}}=0, \\
\nu \frac{\partial \phi}{\partial y}-2 i \tau \nu \frac{\partial \phi}{\partial x}-\nu^{2} \phi+\tau^{2} \frac{\partial^{2} \phi}{\partial x^{2}}=0, \quad y=0, \\
\lim _{x \rightarrow \infty}\left[\left|\frac{\partial \phi}{\partial x}\right|^{2}+\left|\frac{\partial \phi}{\partial y}\right|^{2}\right]=0,
\end{gathered}
$$

where

$$
\tau=\omega c / g, \quad \nu=\omega^{2} / g .
$$

(I) Pulsating moving source. Writing the space potential of our moving source (located at $\zeta=\xi+i \eta$ ) in the form

$$
G_{1}=\ln |z-\zeta|-\ln |z-\bar{\zeta}|+G(x, y ; \xi, \eta),
$$

where $G(x, y ; \xi, \eta)$ is harmonic in the lower half plane, and noting that at $y=0$ :

$$
\begin{gathered}
\frac{\partial}{\partial y} \ln |z-\zeta|=-\frac{\partial}{\partial y} \ln |z-\bar{\zeta}|=g(z-\bar{\zeta})^{-1}, \\
\frac{\partial^{n}}{\partial x^{n}} \ln |z-\zeta|=\frac{\partial^{n}}{\partial x^{n}} \ln |z-\bar{\zeta}|,
\end{gathered}
$$

it is easy to see that $G$ must satisfy the following differential equation at $y=0$ :

$$
\nu \frac{\partial G}{\partial y}-2 i \tau \nu \frac{\partial G}{\partial x}-\nu^{2} G+\tau^{2} \frac{\partial^{2} G}{\partial x^{2}}=2 \nu \frac{\partial}{\partial y} \ln |z-\bar{\zeta}|
$$

Now both sides of (7) involve functions harmonic in the lower half plane; (7) holds for entire lower half plane by analytic extension. 
From the well-known relation

$$
(z-\bar{\zeta})^{-1}=i \int_{0}^{\infty} \exp [-i k(z-\bar{\zeta})] d k, \quad y+\eta<0,
$$

the following expression can easily be established:

$2 \nu \frac{\partial}{\partial y} \ln |z-\bar{\zeta}|=-\nu \int_{0}^{\infty} \exp [k(y+\eta)]\{\exp [i k(x-\xi)]+\exp [-i k(x-\xi)]\} d k$.

Thus combining (9) and (7), one obtains

$$
\begin{aligned}
G=\nu \int_{0}^{\infty} \frac{\exp [k(y+\eta)]}{\tau^{2} k^{2}+(2 \tau-1) \nu k+\nu^{2}} & \exp [-i k(x-\xi)] d k \\
& +\nu \int_{0}^{\infty} \frac{\exp [k(y+\eta)]}{\tau^{2} k^{2}-(2 \tau+1) \nu k+\nu^{2}} \exp [i k(x-\xi)] d k
\end{aligned}
$$

The disturbance velocity potential of a moving pulsating source can therefore be written in the following form

$$
\begin{aligned}
\Phi= & \{\ln |z-\zeta|-\ln |z-\bar{\zeta}| \\
& \left.\quad+G+\sum C_{n} \exp \left[k_{n}(y+\eta)\right] \exp \left[ \pm i k_{n}(x-\xi)\right]\right\} \exp (i \omega t) \\
= & G^{*}(z, \zeta) \exp (i \omega t),
\end{aligned}
$$

where the $k_{n}$ 's are the poles of the integrands in (10). The constants $C_{n}$ are to be determined by the physical condition at upstream infinity (3), i.e.

$$
\lim _{x \rightarrow \infty}\left[\left|\frac{\partial G^{*}}{\partial x}\right|^{2}+\left|\frac{\partial G^{*}}{\partial y}\right|^{2}\right]=0 .
$$

(II) Fluctuating moving vortex. Analogous to (I), one can write the space potential of our single moving vortex (located at $\zeta=\xi+i \eta$ ) in the form

$$
H_{1}=\theta(z-\zeta)+\theta(z-\bar{\zeta})+H(x, y ; \xi, \eta),
$$

where $H(x, y ; \xi, \eta)$ is harmonic in the lower half plane. Noting the symmetry of $\theta+\bar{\theta}$, $(\bar{\theta}=\theta(z-\bar{\zeta}))$, at $y=0$, i.e.

$$
\begin{aligned}
& \frac{\partial}{\partial y} \theta=\frac{\partial}{\partial y} \bar{\theta}=R(z-\bar{\zeta})^{-1}, \\
& \frac{\partial^{n}}{\partial x^{n}} \theta=-\frac{\partial^{n}}{\partial x^{n}} \bar{\theta}
\end{aligned}
$$

it can easily be shown that $H$ must satisfy the differential equation

$$
\nu \frac{\partial H}{\partial y}-2 i \tau \nu \frac{\partial H}{\partial x}-\nu^{2} H+\tau^{2} \frac{\partial^{2} H}{\partial x^{2}}=-2 \nu \frac{\partial}{\partial y} \bar{\theta} .
$$

Now

$$
2 \nu \frac{\partial \bar{\theta}}{\partial y}=i \nu \int_{0}^{\infty} \exp [k(y+\eta)][\exp [i k(x-\xi)]-\exp [-i k(x-\xi)]] d k
$$


Thus combining (16) and (15), one obtains

$$
\begin{aligned}
H=i \nu \int_{0}^{\infty} \frac{\exp [k(y+\eta)]}{\tau^{2} k^{2}-(2 \tau+1) \nu k+\nu^{2}} \exp [i k(x-\xi)] d k \\
\quad-i \nu \int_{0}^{\infty} \frac{\exp [k(y+\eta)]}{\tau^{2} k^{2}+(2 \tau-1) \nu k+\nu^{2}} \exp [-i k(x-\xi)] d k .
\end{aligned}
$$

The disturbance velocity potential of a moving vortex of fluctuating strength accordingly can be written

$$
\begin{aligned}
\Phi_{1} & =\left\{\theta+\bar{\theta}+H+\sum D_{n} \exp \left[k_{n}(y+\eta)\right] \exp \left[ \pm i k_{n}(x-\xi)\right]\right\} \exp (i \omega t) \\
& =H^{*}(z, \zeta) \exp (i \omega t)
\end{aligned}
$$

where, as before, the $k_{n}$ 's are the poles of the integrands in (17), and the constants $\left.I\right)_{n}$ are to be determined by the physical condition at upstream infinity, Eq. (3).

It should be noted, however, that fluctuation in strength of a single travelling vortex does not occur alone, i.e., this fluctuation is necessarily accompanied by a simultaneous shedding of vortices into the wake, of an amount equal and opposite to the increment in travelling vortex strength, so that Helmholtz's law is not violated. Expression (18) accordingly represents only a part of the complete velocity potential of the induced flow field. The complete potential is obtained by simply adding to (18) the contribution due to the distributed wake vortices. Noting that

$$
\frac{\partial \Phi_{1}}{\partial t^{\prime}}=i \omega H^{*}\left(z, \zeta^{\prime}\right) \exp \left[i \omega\left(t+t^{\prime}\right)\right]
$$

and

$$
c t^{\prime}=\xi^{\prime}-\xi, \quad c d t^{\prime}=d \xi^{\prime},
$$

where the primed system denotes the running coordinate, it can easily be shown that the latter contribution is given by the following integration

$$
\Phi^{\prime}=-\int_{-\infty}^{0} \frac{\partial \Phi_{1}}{\partial t^{\prime}} d t^{\prime}=-\frac{i \omega}{c} \int_{-\infty}^{\xi} H^{*}\left(z, \zeta^{\prime}\right) \exp \left\{i \omega\left[t-\frac{1}{c}\left(\xi-\xi^{\prime}\right)\right]\right\} d \xi^{\prime} .
$$

Hence the complete velocity potential of a single vortex of fluctuating strength travelling beneath a free surface can be written in the following form:

$$
\Phi=\left[H^{*}(z, \zeta)-\frac{i \omega}{c} \int_{-\infty}^{\xi} H^{*}\left(z, \zeta^{\prime}\right) \exp \left[-\frac{i \omega}{c}\left(\xi-\xi^{\prime}\right)\right] d \xi^{\prime}\right] \exp (i \omega t)
$$

It might be of interest to remark that a travelling vortex line of fluctuating strength is an idealization of a lifting surface with oscillating angle of attack, thus (20) actually offers a first approximation to the solution of the oscillating hydrofoil. An exact solution to the oscillating hydrofoil requires, of course, the determination of the distribution of fundamental solutions (11) and (20) along the foil contour so that the boundary condition on contour and Kutta condition at the trailing edge be satisfied. In that case it will not be difficult to see that the problem leads directly to a Fredholm type integral equation of the second kind. 